\title{
Effects of triptolide on the expression of MHC II in microglia in kainic acid-induced epilepsy
}

\author{
ZHENG SUN ${ }^{1,2^{*}}$, MENG DU $^{1 *}$, YAO LU ${ }^{3}$ and CHANG-QIAN ZENG ${ }^{1}$ \\ ${ }^{1}$ Medical College, Dalian University, Dalian, Liaoning 116622; ${ }^{2}$ Beijing International Travel Health Care Center of \\ Beijing Entry-Exit Inspection and Quarantine Bureau, Beijing 100088; ${ }^{3}$ Neonatal Screening Center, \\ Maternal and Child Health Care Hospital of Dalian, Dalian, Liaoning 116033, P.R. China
}

Received June 29, 2017; Accepted November 17, 2017

DOI: $10.3892 / \mathrm{mmr} .2018 .8891$

\begin{abstract}
The purpose of the present study was to determine whether triptolide (T10) had any effect on major histocompatibility complex class II (MHC II) expression in kainic acid (KA)-activated microglia, and to investigate the underlying molecular mechanism. BV-2 microglia were pretreated with T10 prior to activation with KA. The expression level of MHC II and class II transactivator (CIITA) mRNA was determined via reverse transcription-polymerase chain reaction. The expression of MHC II, CIITA and the phosphorylation level of c-Jun and proto-oncogene c-Fos (c-Fos) was determined by western blotting. The protein expression level of MHC II was determined by immunocytochemistry. It was observed that the mRNA and protein levels of MHC II and CIITA were increased in KA-activated BV-2 microglia, and that this increase was almost completely eliminated by T10. AP-1 is a family of homodimers or heterodimers, composed of Jun family and Fos family proteins. Sequence analysis revealed an AP-1 DNA binding site in the promoter of CIITA. The phosphorylation of c-Jun and c-Fos was increased in KA-activated microglia, while T10 was able to suppress the phosphorylation of c-Jun and c-Fos in KA-activated microglia. These data suggested that T10 may exert suppressive effects on MHC II expression in KA-activated microglia, and that the mechanism may involve the regulation of AP-1 activity.
\end{abstract}

\section{Introduction}

Epilepsy, the 3rd most common chronic brain disorder, is characterized by an enduring predisposition to seizures, and by emotional and cognitive dysfunction (1). Microglia serve

Correspondence to: Professor Chang-Qian Zeng, Medical College, Dalian University, 10 University Avenue, Dalian, Liaoning 116622, P.R. China

E-mail: zengchangqian@163.com

${ }^{*}$ Contributed equally

Key words: triptolide, BV-2 microglia, major histocompatibility complex class II, class II transactivator, transcription factor AP-1, kainic acid an important role in epilepsy and the pathological hallmark of epilepsy is an increase in the number of microglia and a decrease in neurons (2).

Major histocompatibility complex class II (MHC II) expression serves a critical role in the induction of immune responses through presentation of antigenic peptides to $\mathrm{CD}^{+}$ $\mathrm{T}$ lymphocytes (3). Constitutive expression is restricted to a limited number of professional antigen-presenting cells, although a variety of cell types express MHC II following stimulation by interferon- $\gamma(4,5)$.

Microglia cells, as the resident innate immune cells of the central nervous system (CNS), generally express low levels of MHC II proteins; however, in inflammatory or neurodegenerative conditions, activated microglial cells exhibit highly upregulated MHC II expression (6-8). Microglia with high expression of MHC II may activate $\mathrm{CD}^{+} \mathrm{T}$ lymphocytes, and induce immune responses that may act by inducing neuronal cell death or the degeneration of neuronal processes $(9,10)$. In epileptic rat models, MHC II-expressing microglia were associated with neuronal death processes (11). Therefore, the inhibition of microglial cell activation and MHC II expression may be therapeutically significant in epilepsy.

Tripterygium wilfordii Hook F (TWHF), a member of the Celastraceae plant family, has been identified to have potent anti-inflammatory and immunosuppressive functions, and is widely used in China for the treatment of rheumatoid arthritis and systemic lupus erythematosus (12). Triptolide (designated as T10) is one of the major active ingredients of TWHF that performs anti-inflammatory and immunosuppressive functions $(13,14)$. Due to its small molecular size (molecular weight, $360 \mathrm{~g} / \mathrm{mol}$ ) and lipophilic properties, T10 is able to penetrate the blood-brain barrier readily, making it a potential neuroprotective drug for the treatment of CNS inflammatory diseases. Previously, T10 has been demonstrated to be beneficial in animal models of numerous CNS disorders, including Alzheimer's and Parkinson's diseases $(15,16)$.

A previous study demonstrated that the administration of T10 markedly alleviated seizure behavior and prevented damage to neurons in epileptic rat models. It was additionally observed that the expression of MHC II on microglia in the hippocampi of epileptic rats induced by kainic acid (KA) was markedly decreased by treatment with T10 at a dose of $30 \mu \mathrm{g} / \mathrm{kg}$ (17). However, the precise molecular mechanisms through which T10 
may affect MHC II expression remain unknown. Understanding the mechanism of the repressive effect of T10 on MHC II expression may elucidate important pathways that may be targeted to treat epilepsy. Therefore, in the present study, the molecular mechanisms underlying the effect of T10 on MHC II expression in KA-activated microglia were investigated.

\section{Materials and methods}

Materials. Dulbecco's modified Eagle's medium (DMEM) was purchased from Gibco (Thermo Fisher Scientific, Inc., Waltham, MA, USA). Fetal bovine serum (FBS) was purchased from Hyclone (GE Healthcare Life Sciences, Logan, UT, USA). KA was purchased from Sigma-Aldrich (Merck KGaA, Darmstadt, Germany) and T10 was purchased from Shenyang Longpu Technology. Co., Ltd. (Shenyang, China). TRIzol reagent was purchased from Invitrogen (Thermo Fisher Scientific, Inc.). The PrimeScript Reverse Transcriptase kit and RNA Polymerase Chain Reaction (PCR) kit version 3.0 were purchased from Takara Biotechnology Co., Ltd. (Dalian, China). Rat monoclonal anti-mouse MHC II antibody (cat. no. SC-59322) and rabbit polyclonal anti-mouse CIITA antibody (cat. no. SC-48797) were purchased from Santa Cruz Biotechnology, Inc. (Dallas, TX, USA). Rabbit monoclonal anti-mouse phosphorylated c-Fos (cat. no. 5348), rabbit polyclonal anti-mouse phosphorylated c-Jun (cat. no. 9164), rabbit anti-mouse $\beta$-actin (cat. no. 4970), goat anti-rat IgG-HRP second antibody (cat. no. 7077) and goat anti-rabbit IgG-HRP second antibody (cat. no. 7074) were purchased from Cell Signaling Technology, Inc. (Danvers, MA, USA). Radioimmunoprecipitation assay (RIPA) lysis buffer was purchased from Nanjing KeyGen Biotech Co., Ltd. (Nanjing, China).

BV-2 microglia culture. The BV-2 microglia cell line was provided by Professor Jinyan Wang (Chinese Medical University, Liaoning, China) and were maintained in DMEM supplemented with $10 \%$ FBS, $100 \mathrm{U} / \mathrm{ml}$ penicillin, and $100 \mu \mathrm{g} / \mathrm{ml}$ streptomycin. BV-2 microglia were kept in a humidified incubator with $5 \% \mathrm{CO}_{2}$ at $37^{\circ} \mathrm{C}$. The cells were passaged every 3 days while growing to $75-80 \%$ confluence.

$B V-2$ microglia treatment. T10 was dissolved in dimethyl sulfoxide and mixed with culture medium to a concentration of $10 \mathrm{nM}$. KA was diluted in culture medium to a concentration of $100 \mu \mathrm{M}$. For treatment with KA, BV-2 microglia cells were stimulated with KA for $2 \mathrm{~h}$. For treatment with T10, BV-2 microglia were pretreated with $\mathrm{T} 10$ for $16 \mathrm{~h}$ prior to stimulation with KA. BV-2 microglia cultured in DMEM without any treatment served as controls.

Immunocytochemistry. BV-2 microglia were treated as described above. Cells were fixed with cold $4 \%$ paraformaldehyde for $15 \mathrm{~min}$ and washed in PBS three times for $5 \mathrm{~min}$ followed by incubation with normal goat serum (Beyotime Institute of Biotechnology, Shanghai, China) for $20 \mathrm{~min}$. Cells were subsequently incubated with MHC II primary antibody (1:300) overnight at $4^{\circ} \mathrm{C}$, followed by incubation with goat anti-rat IgG-HRP secondary antibody $(1: 1,000)$ for $30 \mathrm{~min}$ at room temperature. Color was developed with 3,3'-diaminobenzidine for $15 \mathrm{~min}$ at room temperature and cells were observed
Table I. Sequences of primers used for reverse transcription-polymerase chain reaction analysis.

\begin{tabular}{ll}
\hline Genes & \multicolumn{1}{c}{ Primer sequence (5'-3') } \\
\hline MHC II & F: GGACCCCACAGGACTTCACATACT \\
& R: GCCGTCTTCTCCTTGTTGCTGTGG \\
CIITA & F: TGC AGGCGACCAGGAGAG ACA \\
& R: GAA GCT GGG CAC CTC AAAGAT \\
$\beta$-actin & F: TGGAATCCTGTGGCATCCATGAAAC \\
& R: TAAAACGCAGCTCAGTAACAGTCCG
\end{tabular}

MHC II, major histocompatibility complex class II; CIITA, class II transactivator; F, forward; R, reverse.

with an inverted microscope at x200 magnification (CKX41; Olympus Corporation, Tokyo, Japan).

Isolation of total RNA and reverse transcription (RT)-PCR. Total RNA was prepared using TRIzol reagent and primed with random hexamers for the synthesis of complementary DNA using Avian Myeloblastosis Virus Reverse Transcriptase (Takara Biotechnology Co., Ltd., Dalian, China), according to the manufacturer's instructions using DNAse-pretreated total mRNA. Single-stranded cDNA was amplified via PCR with primers for MHC II, CIITA and $\beta$-actin (Table I). The product lengths for MHC II, CIITA and $\beta$-actin were 394, 489 and 349 bp, respectively. The following PCR conditions were applied: 35 cycles at $94^{\circ} \mathrm{C}$ for $30 \mathrm{sec} ; 54^{\circ} \mathrm{C}$ (MHC II), $58^{\circ} \mathrm{C}$ (CIITA) or $53^{\circ} \mathrm{C}\left(\beta\right.$-actin) for $30 \mathrm{sec}$; and $72^{\circ} \mathrm{C}$ for $1 \mathrm{~min}$. $\beta$-actin was used as an internal control to evaluate the relative expression of MHC II and CIITA. The PCR products were separated on a $1 \%$ agarose gel and visualized under ultraviolet light following staining with GoldViewand the results were analyzed by Quantity-One software (version 4.62; Bio-Rad Laboratories, Inc., Hercules, CA, USA).

Promoter prediction. The CIITA gene promoter was predicted by PROSCAN version 1.7 software (https://www-bimas.cit. nih.gov/molbio/proscan/).

Western blotting. BV-2 microglia were washed with PBS three times, placed at a temperature of $4^{\circ} \mathrm{C}$, and lysed for $30 \mathrm{~min}$ in RIPA lysis buffer. Lysates were subsequently centrifuged at $800 \mathrm{x} \mathrm{g}$ for $20 \mathrm{~min}$ at $4^{\circ} \mathrm{C}$. Protein concentration was quantified using the Bradford assay with the Bio-Rad Protein Assay (Bio-Rad Laboratories, Inc.) according to the manufacturer's instructions. Equal amounts of protein $(60-80 \mu \mathrm{g})$ were separated electrophoretically using SDS-PAGE on a $10 \%$ gel; the gel was subsequently transferred to $0.45-\mu \mathrm{m}$ polyvinylidene fluoride membranes. Membranes were soaked in 5\% bovine serum albumin (Sigma Chemical Co., St. Louis, MO, USA) for $2 \mathrm{~h}$ at room temperature, followed by incubation with the appropriate primary antibodies (MHC II and CIITA at 1:500; p-c-Fos, p-c-Jun and $\beta$-actin at 1:1,000) overnight at $4^{\circ} \mathrm{C}$. Following washing with TBS-Tween-20, the membranes were incubated with the appropriate secondary peroxidase-conjugated antibodies $(1: 1,000)$ for $2 \mathrm{~h}$ at room temperature, 

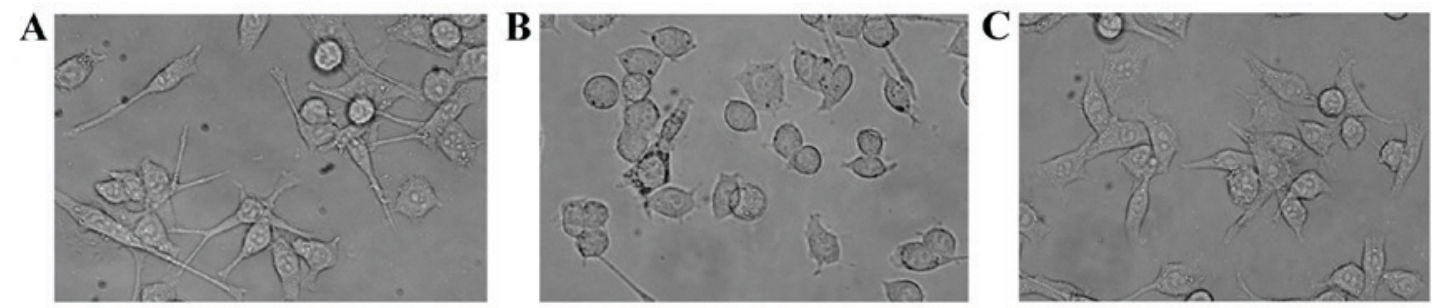

Figure 1. Effect of T10 on the morphology of KA-activated microglia. (A) Normal BV-2 microglia; (B) BV-2 microglia treated with $100 \mu \mathrm{M} \mathrm{KA}$ for $2 \mathrm{~h}$; and (C) BV-2 microglia pretreated with $10 \mathrm{nM}$ T10 for $16 \mathrm{~h}$ prior to treatment with KA. Magnification, $\mathrm{x} 400$. KA, kainic acid; T10, triptolide.
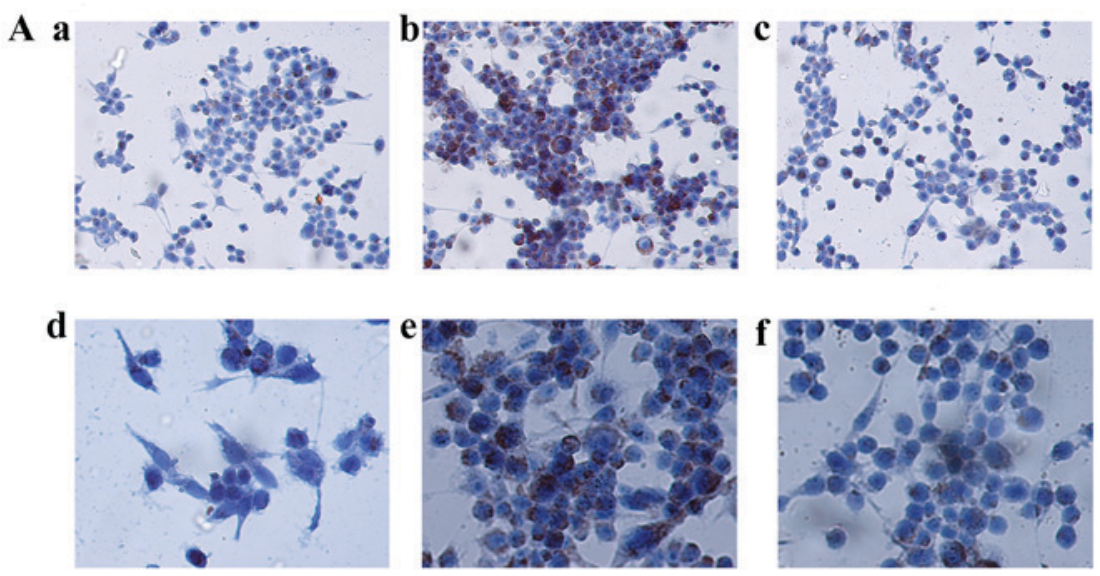

B
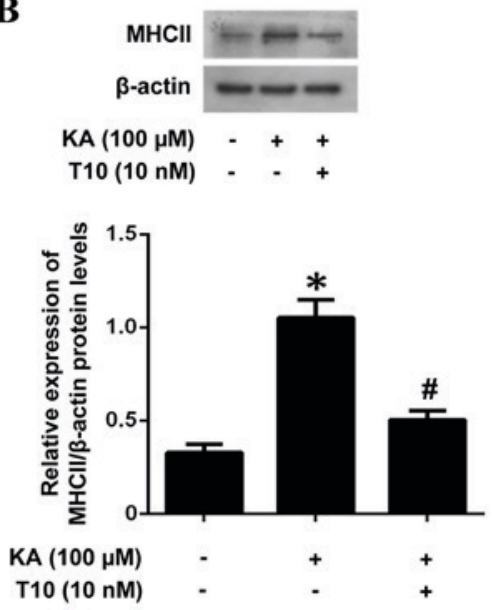

C
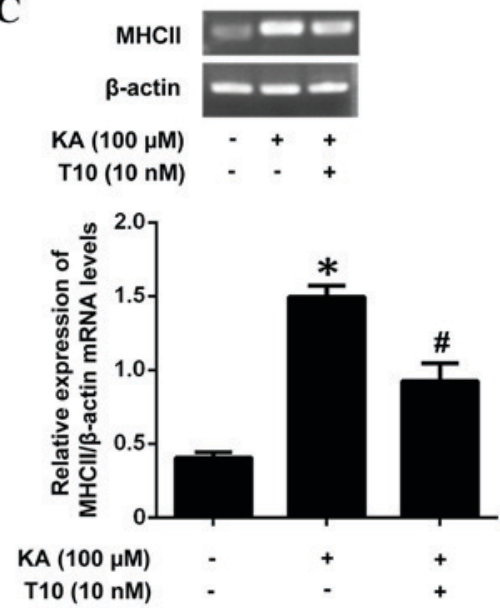

Figure 2. Effect of T10 on the MHC II protein and mRNA expression levels in KA-activated microglia. The expression of MHC II protein was determined by (A) immunocytochemistry: (a) Normal BV-2 microglia at x200 magnification; (b) BV-2 microglia treated with $100 \mu \mathrm{m} \mathrm{KA}$ for $2 \mathrm{~h}$, at x 200 magnification; (c) BV-2 microglia pretreated with $10 \mathrm{nM}$ T10 for $16 \mathrm{~h}$ prior to treatment with KA, at x200 magnification; (d) normal BV-2 microglia at x400 magnification; (e) KA-treated BV-2 microglia at x400 magnification; and (f) T10-pretreated microglia treated with KA, at x400 magnification. (B) Western blotting was additionally used to measure the protein expression levels of MHC II. The levels of MHC II mRNA were determined by (C) reverse transcription-polymerase chain reaction. Values are expressed as the mean \pm standard deviation of three separate experiments. ${ }^{*} \mathrm{P}<0.05$ vs. control group; ${ }^{\text {} P}<0.05$ vs. KA group. KA, kainic acid; T10, triptolide; MHC II, major histocompatibility complex class II.

and visualized using an enhanced chemiluminescence kit (Beyotime Institute of Biotechnology,). Images were captured and analyzed using a ChemiDoc ${ }^{\mathrm{TM}} \mathrm{XRS}+$ imaging system (Bio Rad Laboratories, Inc.).

Statistical analysis. All statistics were calculated using SPSS 2.0 (SPSS, Inc., Chicago, IL, USA). One-way analysis of variance followed by Fisher's least significant difference post hoc test was used to calculate the statistical differences between the control and treated samples. Values are expressed as the mean \pm standard deviation of three separate experiments. $\mathrm{P}<0.05$ was considered to indicate a statistically significant difference.

\section{Results}

Effect of T10 on the morphology of KA-activated microglia. Under normal conditions, BV-2 microglia remain in a resting state and, morphologically, appear to be non-ramified cells (Fig. 1A). Following treatment with KA $(100 \mu \mathrm{M})$ for $2 \mathrm{~h}, \mathrm{BV}-2$ microglia exhibited a highly differentiated state with enlarged 
A

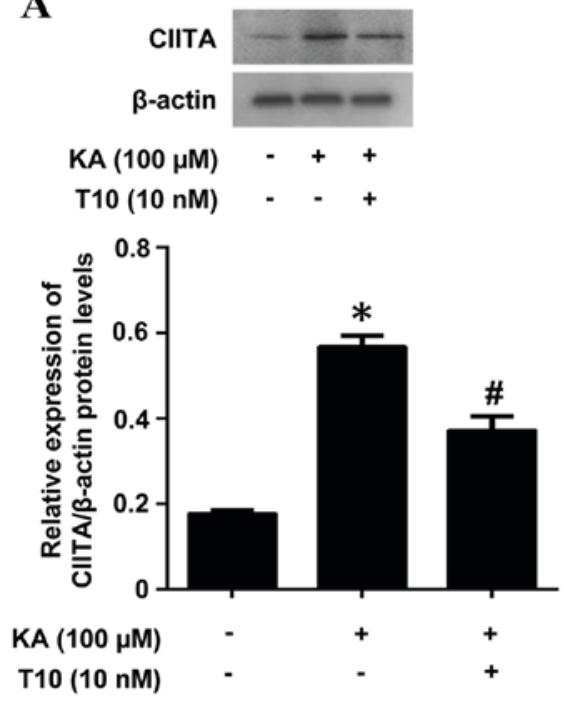

B
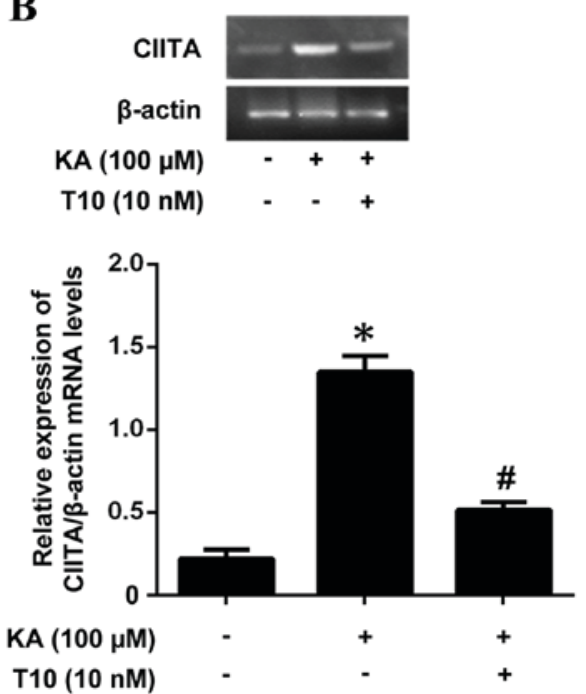

Figure 3. Effect of T10 on the CIITA protein and mRNA expression level in KA-activated microglia. The expression of CIITA protein and mRNA was determined by (A) western blotting and (B) reverse transcription-polymerase chain reaction. Values are expressed as the mean \pm standard deviation of three separate experiments. "P<0.05 vs. control group; ${ }^{\text {}} \mathrm{P}<0.05$ vs. KA group. KA, kainic acid; T10, triptolide; CIITA, class II transactivator.

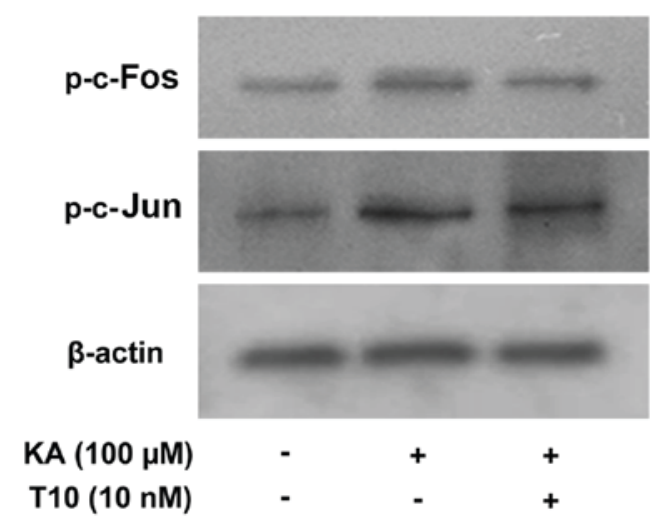

Figure 4. T10 decreases the increased expression of p-c-Fos and p-c-Jun in KA-activated microglia. The expression of p-c-Fos, p-c-Jun proteins were determined by western blotting. T10, triptolide; p, phosphorylated; c-Fos, proto-oncogene c-Fos; KA, kainic acid.

cell bodies (Fig. 1B). Treatment with T10 markedly inhibited the activation of microglia (Fig. 1C).

Effect of T10 on MHC II $m R N A$ and protein expression in $K A$-activated microglia. In order to investigate the inhibitory effect of T10 on MHC II mRNA and protein levels, BV-2 microglia were pretreated with T10 $(10 \mathrm{nM})$ for $16 \mathrm{~h}$ prior to stimulation with KA for $2 \mathrm{~h}$. The pretreatment with T10 significantly inhibited KA-induced MHC II protein expression (Fig. 2A and B). The results were additionally confirmed by RT-PCR (Fig. 2C), which illustrated an increased expression level of MHC II in KA-treated microglia and a decreased expression level of MHC II in microglia pretreated with $10 \mathrm{nM}$ $\mathrm{T} 10$ for $16 \mathrm{~h}$. The results indicated that T10 may significantly decrease the mRNA and protein expression of MHC II in KA-activated microglia.

Effects of T10 on the expression of CIITA mRNA and protein in KA-activated $B V$-2 microglia. As CIITA is a key factor in
MHC II expression, the present study investigated the ability of T10 to affect CIITA expression as a potential mechanism of the inhibitory effects of T10 on MHC II expression. The mRNA and protein levels of CIITA were measured by RT-PCR and western blotting. Pretreatment with T10 significantly inhibited the KA-induced CIITA protein expression (Fig. 3A). The results were additionally confirmed by RT-PCR (Fig. 3B), which exhibited an increased level of CIITA in KA-treated microglia, and a decreased level of CIITA in microglia pretreated with $10 \mathrm{nM} \mathrm{T10}$ for $16 \mathrm{~h}$. The results suggested that T10 may target signaling pathways involved in CIITA expression in KA-activated BV-2 microglia.

AP-1 binding sites are present at the CIITA promoter. PROSCAN prediction of the CIITA promoter highlighted to the presence of AP-1 binding sites at positions -9113/-9105 from the site of transcription initiation.

Effects of T10 on the phosphorylation of c-Jun and c-Fos in KA-activated BV-2 microglia. In order to determine the effect of T10 on the activation of AP-1, its effects on the phosphorylation of c-Jun and c-Fos were investigated. The level of phosphorylated c-Jun and c-Fos was measured by western blotting (Fig. 4). KA markedly increased the level of phosphorylated c-Jun and c-Fos expression. Pretreatment with T10 decreased the increased expression of p-c-Jun and p-c-Fos in KA-activated BV-2 microglia. The results indicated that T10 may modulate AP-1 activity.

\section{Discussion}

Under normal conditions, microglia, as the resident macrophages in the brain, remain in a less immunoreactive state and perform immune surveillance functions (18). In response to abnormal stimulation, microglia become activated very rapidly. The activated microglia are differentiated into macrophage-like and dendritic-like cells, exhibiting upregulated expression 
levels of MHC II and co-stimulatory proteins that are required for antigen presentation to T cells (19). In tetanus toxin-induced epileptic rat models, MHC II-expressing microglia are located in the dorsal hippocampi of rats exhibiting cell loss in the CA1 region (11). In the present study, BV-2 microglia exhibited morphological alterations and became activated microglia following treatment with KA for $2 \mathrm{~h}$. The results demonstrated that the mRNA and protein expression levels of MHC II were increased in KA-activated BV-2 microglia. It has been reported that T10 possesses potent anti-inflammatory and immunosuppressive effects. A previous study demonstrated that T10 significantly inhibited proinflammatory factor-induced overexpression of MHC II and B7 molecules in renal tubular epithelial cells (20). In the present study, T10 markedly blocked the activation of microglia and inhibited MHC II expression in KA-activated microglia.

The regulation of MHC II genes occurs primarily at the transcriptional level, and the non-DNA-binding protein CIITA has been demonstrated to be the master transactivator for class II transcription. CIITA is essential, and is the major rate-limiting factor for inducible and constitutive MHC II expression $(21,22)$. CIITA-deficient mice lack inducible MHC II expression and exhibit sparse constitutive MHC II expression on subsets of thymic stromal cells (23). Nikodemova et al (24) reported that minocycline was able to significantly decrease the severity of the clinical course of experimental allergic encephalomyelitis; the molecular mechanisms of action of minocycline may involve the decreased expression of CIITA and result in a decrease in MHC II expression in microglia. The results of the present study demonstrated that $\mathrm{T} 10$ inhibited the expression of CIITA in KA-activated microglia. The results of the present study indicated that the inhibitory actions of T10 on MHC II overexpression occur at the transcriptional level.

Mitogen-activated protein kinases (MAPKs) are the most important signaling molecules with involvement in activated microglia (25). MAPKs directly regulate downstream targets via phosphorylation, and AP-1 is one of the downstream targets. AP-1 is a transcription factor complex composed of Jun family homodimers (c-Jun, Jun D and Jun B) or heterodimers composed of Jun family members with any of the Fos family members (c-Fos, Fos B, Fra1 and Fra2). Following phosphorylation, AP-1 translocates to the nucleus and binds to specific response elements on downstream target genes which, in turn, promote immediate early gene expression. A previous study established that MAPK-extracellular signal-regulated kinase and MAPK8 are required for the regulation of CIITA and MHC II expression in melanoma cell lines through an AP-1-responsive motif in CIITA promoter III (26). PROSCAN CIITA promoter analysis highlighted to the presence of AP-1 binding sites at positions -9113/-9105 from the site of transcription initiation. Thus, it was hypothesized that AP-1 may be involved in regulating CIITA and subsequent MHC II expression. In the present study, T10 decreased the phosphorylation of c-Jun and c-Fos, which may be the molecular mechanism through which $\mathrm{T} 10$ reduces the expression of CIITA.

In conclusion, the results of the present study indicated that T10 significantly decreased the expression of MHC II in KA-activated BV-2 microglia. The underlying mechanism may involve the inhibition of CIITA expression via a mechanism involving the inhibition of AP-1 activation. These results point to a potential regulatory mechanism of MHC II expression in KA-activated microglia, mediated by T10. T10 may be useful in the treatment of epilepsy and other neurodegenerative diseases that are characterized by overexpression of MHC II.

\section{Acknowledgements}

The authors of the present study would like to thank Professor Jinyan Wang (Chinese Medical Sciences University, Liaoning, China) for providing the BV-2 microglia.

\section{References}

1. Duncan JS, Sander JW, Sisodiya SM and Walker MC: Adult epilepsy. Lancet 367: 1087-1100, 2006.

2. Tegelberg S, Kopra O, Joensuu T, Cooper JD and Lehesjoki AE: Early microglial activation precedes neuronal loss in the brain of the $\mathrm{Cstb}^{--}$mouse model of progressive myoclonus epilepsy, EPM1. J Neuropathol Exp Neurol 71: 40-53, 2012.

3. de Graaf KL, Barth S, Herrmann MM, Storch MK, Otto C, Olsson T, Melms A, Jung G, Wiesmüller KH and Weissert R: MHC class II isotype- and allele-specific attenuation of experimental autoimmune encephalomyelitis. J Immunol 173: 2792-2802, 2004.

4. Casas C, Herrandograbulosa M, Manzano R, Mancuso R and Osta R, Navarro X: Early presymptomatic cholinergic dysfunction in a murine model of amyotrophic lateral sclerosis. Brain Behav 3: 145-158, 2013.

5. Vardjan N, Gabrijel M, Potokar M, Svajger U, Kreft M, Jeras M, de Pablo Y, Faiz M, Pekny M and Zorec R: IFN- $\gamma$-induced increase in the mobility of MHC class II compartments in astrocytes depends on intermediate filaments. J Neuroinflammation 9: 144, 2012.

6. Beach TG, Woodhurst WB, Macdonald DB and Jones MW: Reactive microglia in hippocampal sclerosis associated with human temporal lobe epilepsy. Neurosci Lett 191: 27-30, 1995.

7. Depboylu C, Stricker S, Ghobril JP, Oertel WH, Priller J and Höglinger GU: Brain-resident microglia predominate over infiltrating myeloid cells in activation, phagocytosis and interaction with T-lymphocytes in the MPTP mouse model of Parkinson disease. Exp Neurol 238: 183-191, 2012.

8. Wojtera M, Sobów T, Kłoszewska I, Liberski PP, Brown DR and Sikorska B: Expression of immunohistochemical markers on microglia in Creutzfeldt-Jakob disease and Alzheimer's disease: Morphometric study and review of the literature. Folia Neuropathol 50: 74-84, 2012.

9. Marinova-Mutafchieva L, Sadeghian M, Broom L, Davis JB, Medhurst AD and Dexter DT: Relationship between microglial activation and dopaminergic neuronal loss in the substantia nigra: A time course study in a 6-hydroxydopamine model of Parkinson's disease. J Neurochem 110: 966-975, 2009.

10. Nakahara H, Konishi Y, Beach TG, Yamada N, Makino S and Tooyama I: Infiltration of $\mathrm{T}$ lymphocytes and expression of icam-1 in the hippocampus of patients with hippocampal sclerosis. Acta Histochem Cytochem 43: 157-162, 2010.

11. Shaw JA, Perry VH and Mellanby J: MHC class II expression by microglia in tetanus toxin-induced experimental epilepsy in the rat. Neuropathol Appl Neurobiol 20: 392-398, 1994.

12. Tao X, Cush JJ, Garret M and Lipsky PE: A phase I study of ethyl acetate extract of the chinese antirheumatic herb Tripterygium wilfordii hook $\mathrm{F}$ in rheumatoid arthritis. J Rheumatol 28: 2160-2167, 2001.

13. Du F, Liu T, Liu T, Wang Y, Wan Y and Xing J: Metabolite identification of triptolide by data-dependent accurate mass spectrometric analysis in combination with online hydrogen/deuterium exchange and multiple data-mining techniques. Rapid Commun Mass Spectrom 25: 3167-3177, 2011.

14. Gu WZ, Chen R, Brandwein S, Mcalpine J and Burres N: Isolation, purification, and characterization of immunosuppressive compounds from tripterygium: Triptolide and tripdiolide. Int J Immunopharmacol 17: 351-356, 1995.

15. Li FQ, Lu XZ, Liang XB, Zhou HF, Xue B, Liu XY, Niu DB, Han JS and Wang XM: Triptolide, a Chinese herbal extract, protects dopaminergic neurons from inflammation-mediated damage through inhibition of microglial activation. J Neuroimmunol 148: 24-31, 2004. 
16. Jiao J, Xue B, Zhang L, Gong Y, Li K, Wang H, Jing L, Xie J and Wang $X$ : Triptolide inhibits amyloid-beta1-42-induced TNF-alpha and IL-1beta production in cultured rat microglia. J Neuroimmunol 205: 32-36, 2008.

17. Lu Y, Sun Z, Zeng CQ and Gao S: Effect of triptolide on the expression of MHC molecules on microglia of kainite-induced rat brain. J Liaoning Univ 14: 112-114, 2012 (In Chinese).

18. Kreutzberg GW: Microglia: A sensor for pathological events in the CNS. Trends Neurosci 19: 312-318, 1996.

19. Ponomarev ED, Novikova M, Maresz K, Shriver LP and Dittel BN: Development of a culture system that supports adult microglial cell proliferation and maintenance in the resting state. J Immunol Methods 300: 32-46, 2005.

20. Li H, Liu ZH, Dai CS, Liu D and Li LS: Triptolide inhibits proinflammatory factor-induced over-expression of class II MHC and B7 molecules in renal tubular epithelial cells. Acta Pharmacol Sin 23: 775-781, 2002

21. Boss JM and Jensen PE: Transcriptional regulation of the MHC class II antigen presentation pathway. Curr Opin Immunol 15: $105-111,2003$
22. Gongora C, Hose S, O'Brien TP and Sinha D: Downregulation of class II transactivator (CIITA) expression by synthetic cannabinoid CP55,940. Immunol Lett 91: 11-16, 2004.

23. Chang $\mathrm{CH}$, Guerder S, Hong SC, van Ewijk W and Flavell RA Mice lacking the MHC class II transactivator (CIITA) show tissue-specific impairment of MHC class II expression. Immunity 4: 167-178, 1996.

24. Nikodemova M, Watters JJ, Jackson SJ, Yang SK and Duncan ID: Minocycline down-regulates MHC II expression in microglia and macrophages through inhibition of IRF-1 and protein kinase $\mathrm{C}$ (PKC)alpha/betaII. J Biol Chem 282: 15208-15216, 2007.

25. Kim SH, Smith CJ and Van Eldik LJ: Importance of MAPK pathways for microglial pro-inflammatory cytokine IL-1 beta production. Neurobiol Aging 25: 431-439, 2004.

26. Martins I, Deshayes F, Baton F, Forget A, Ciechomska I, Sylla K, Aoudjit F, Charron D, Al-Daccak R and Alcaide-Loridan C: Pathologic expression of MHC class II is driven by mitogen-activated protein kinases. Eur J Immunol 37: 788-797, 2007. 